\title{
LA DESCENTRALIZACIÓN EN EL DESARROLLO ECONÓMICO PERUANO
}

\author{
ECONOMIC DEVELOPMENT IN THE PERUVIAN DECENTRALIZATION \\ Abel Andrés Zavala* \\ Docente Auxiliar de la Facultad de Ciencias Económicas \\ Universidad Nacional Mayor de San Marcos-UNMSM / Lima-Perú \\ [Recepción: Marzo de 2014/ Conformidad: Mayo 2014]
}

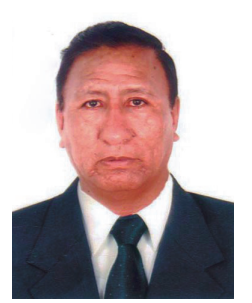

\section{RESUMEN}

El presente artículo aparece en los primeros lugares de la agenda gubernamental. En ese marco, se han adoptado decisiones y políticas públicas que configuran avances importantes, pero aún insuficientes para contrarrestar el perfil centralista tanto de la plataforma productiva como del aparato político administrativo. Mientras este desfase ocurra, se alejarán las posibilidades de satisfacer objetivos de desarrollo equitativo y sostenible para el país en su conjunto.

La reforma o modernización del Estado en general, y del poder ejecutivo en particular, debieran considerar la inserción en la arquitectura gubernamental de estructuras orgánicas y mecanismos de gestión que garanticen la mejor atención de las demandas ciudadanas. Al respecto, tendrán que precisarse con mayor rigor las funciones exclusivas y compartidas de cada nivel de gobierno (nacional, regional, provincial, distrital), teniendo en cuenta que la denominación "regional" es una ficción transitoria de espacios cuya cobertura alude, finalmente a conglomerados departamentales o provinciales aunados por expectativas $y$ potencialidades comunes.

Ha habido un avance en las transferencias de funciones o atribuciones desde el gobierno nacional (ministerios, organismos públicos descentralizados, programas especiales) hacia los gobiernos regionales y municipales. La descentralización en general, es un modelo de desarrollo territorial que satisfaga las legítimas expectativas de inclusión, prosperidad y acceso a mejores oportunidades para el ejercicio de derechos.

\section{Palabras clave:}

Descentralización; centralización; desarrollo económico; estrategia de desarrollo.

\begin{abstract}
This article appears at the top of the government agenda. In this framework, decisions have been taken and policies that shape significant progress, but still not enough to counter the centralized production profile of both platform and the administrative political apparatus. While this mismatch occurs, the chances of meeting goals of equitable and sustainable development for the country as a whole will move away. The reform and modernization of the state in general and the executive in particular should consider the inclusion in the government's organic architecture and mechanisms for management that ensure the best care of citizens' demands structures. In this regard, they have to be specified more rigorously shared at each level of government (national, regional, provincial, district) and exclusive features, considering that the "regional" designation is a transitional spaces fiction whose coverage alluded finally to departmental or provincial conglomerate united by common expectations and potential.

There has been a breakthrough in the transfer of functions or powers from the national government (ministries, decentralized public agencies, special programs) to regional and municipal governments. Decentralization is generally a model of territorial development that meets the legitimate expectations of inclusion, prosperity and access to better opportunities to exercise rights.
\end{abstract}

\section{Keywords:}

Decentralization; centralization; economic development; development strategy.

\footnotetext{
* Economista. Email: aandresz@unmsm.edu.com
} 


\section{INTRODUCCIÓN}

Muchos mitos se han construido para eludir la misión de configurar un Estado y una economía descentralizados, que sean inclusivos y equitativos en la distribución de las oportunidades, de los derechos ciudadanos y de los beneficios generados por el aprovechamiento de nuestros recursos potenciales.

Un primer mito consiste en describir a los procesos descentralistas solo como una lucha por lograr mejores servicios públicos y empleos decentes, entre Lima u otros espacios metropolitanos costeros concentradores del poder y, el resto del país. Esta imagen esconde el hecho de que en esos espacios de poder, se sufre también de cuadros de marginalidad, desesperanza y pobreza, cuyo germen puede encontrarse en una organización del territorio basada en el aprovechamiento depredatorio de materias primas sin mayor valor agregado y en una grave desarticulación entre los mercados a circuitos comerciales internos. Esta percepción equívoca oculta el disloque entre las economías emergentes o modernas y las economías tradicionales, identificadas con los emprendimientos rurales - campesinos empobrecidos, resultante de los todavía ineficaces comportamientos del Estado y de la plataforma económica vigentes para hacer desarrollo descentralizado, equitativo y sostenido.

Otro mito se refiere a la perspectiva sectorial (agricultura, educación, tecnología) con la cual se administran las políticas públicas y las inversiones, lo cual implica desatender la complejidad o multisectorialidad de los procesos de desarrollo. Este mito debe ser desarmado o al menos flexibilizado a partir de un enfoque territorial, que reposa precisamente en entender el desenvolvimiento interdependiente de todos los aspectos que hacen la vida de la gente $y$ de sus instituciones respectivas en determinados espacios locales o regionales. En cualquier caso, esos y otros mitos o desviaciones, deben ser tratados con argumentos razonables para favorecer la implantación de procesos sostenidos de descentralización como referentes funcionales para apostar por el desarrollo en su perspectiva más amplia.

Es en ese inacabable trajinar técnico y político que el presente trabajo, recalca algunos análisis y proposiciones debatidos particularmente en los dos o tres años recientes sobre la cuestión descentralista, en diversos foros realizados en todas las regionales del país. El tema dispone de un conjunto de interpretaciones críticas y propuestas orientadoras que seguramente, como en todo, tendrá vacíos o sesgos temáticos con los cuales no todos estarán conformes. Persigue, en todo caso, mantener latente no solo el discurso pro-descentralista, sino también, fundamentalmente, los retos concretos que siguen pendientes en torno al tratamiento de este crucial tema, tanto por las autoridades como las organizaciones civiles involucradas.

Este trabajo será material de consulta y de trabajo para quienes tienen la responsabilidad de impulsar esta importante política pública, así como, una fuente de información confiable para los actores de la sociedad civil y de la cooperación general.

\section{ORIENTACIÓN POLÍTICAS DE REFORMA DEL ESTADO}

La visión del Estado tiene que revertir la imagen y las características del Estado que nació con la independencia, con miras a lograrlo y plantear a organizar:

\section{Un Estado para todos}

El derecho reclamado puede ser, en el caso peruano, el triunfo electoral, el puesto burocrático o el control de algún recurso clave del poder. Basadre sustenta que en el Perú, es más frecuente el sultanismo o apropiación arbitraria de lo público que el patrimonialismo. Salvo los puestos de confianza que en el Perú, pueden llegar al 5\% de los funcionarios, el patrimonialismo peruano ya no tiene una forma institucional sino que sobrevive como cultura política con miras a controlarlo, es necesario adoptar las siguientes medidas:

- Reducción de los puestos de confianza del 5\% al $3 \%$ en las instituciones públicas.

- Defensa de las instituciones del Estado como espacios del bien común y del interés general.

- Establecimiento de la carrera pública administrativa que separe claramente los puestos de administración de los administradores y que se organice en base al mérito. 


\section{Un Estado soberano}

Se recoge las principales contribuciones de la globalización, pero que defienda el Estado-Nación. En América Latina defendemos el Estado - Nación, para defender la democracia y los derechos de los ciudadanos. Mientras no inventemos una comunidad Sudamérica, la defensa del Estado - Nación sigue siendo una exigencia perentoria como país soberano. Queremos defender nuestros intereses nacionales sin ideologizar las relaciones internacionales. Las siguientes medidas se encaminan en esa dirección:

- La defensa del Estado nacional y de su integridad territorial.

- El reconocimiento de la condición pluricultural del Perú dentro del Estado unitario y descentralizado.

- La participación creativa y competitiva del Perú en el proceso de globalización.

\section{Un Estado moderno}

Es necesario que la mayoría de sus aparatos, especialmente, aquellos que tienen que ver con los servicios y derechos de la población, modernicen la coexistencia de las islas de la modernidad con los aparatos sociales del Estado. Sus aparatos económicos funcionan más o menos bien, pero sus aparatos sociales (salud, educación, seguridad, justicia) dejan mucho que desear, reciben poco presupuesto y son ineficientes. Las siguientes medidas buscan impulsar la modernización del Estado:

-El establecimiento del núcleo estratégico - correspondiente a los tres poderes y al Ministerio Público o sea al gobierno. En sentido alto; que toma todas las decisiones estratégicas, define las leyes y las políticas públicas y exige su cumplimiento en el Poder Ejecutivo. Comprende las actividades del Presidente de la República, de los Ministros, de sus Auxiliares y Asesores Directos.

- El impulso de la especialización funcional de las instituciones estatales para lograr una mayor eficiencia.

- La diferenciación clara de funciones del Estado para definir aquellas que son exclusivas del mismo, aquellas que son compartidas con otros agentes, que pueden ser transferidas a la sociedad civil y que puedan ser tercerizables. Ordenar las competencias y funciones de los sistemas administrativos en los tres niveles de gobierno, articulada al proceso de transferencia de funciones, simplificando su estructura organizacional y mejorando su capacidad de monitoreo.

- Construir el registro unificado de entidades públicas, que permitirá ordenar los sistemas administrativos para contar con un registro común. Construir instrumentos que permitan una gestión más informada, transparente y eficiente, y la construcción de gobiernos abiertos que cuenten con la transparencia, la participación ciudadana y la colaboración de distintos actores del Estado.

- Desarrollar un sistema de seguimiento y evaluación de políticas, que permitirá la retroalimentación de las líneas y objetivos trazados en el plan y la evaluación de los impactos que las acciones generan en el territorio, dada la multiplicidad de objetivos, actores y estrategias.

- Establecer el gobierno electrónico para facilitar el acceso de los ciudadanos a los servicios que brinda el Estado.

-Asimismo, es necesario consolidar las actividades económicas que son exclusivas del Estado. La primera y principal medida es la de garantizar la estabilidad de la moneda. La garantía de estabilidad del sistema financiero es otra actividad estratégica exclusiva del Estado. Esta es la función del Banco Central de Reserva (BCR). Las inversiones en infraestructura y en los servicios públicos no son, en sentido estricto, una actividad exclusiva del Estado, en la medida en que pueden ser objeto de concesión, aunque la responsabilidad por dichas inversiones en este sector sea del Estado.

\section{UN ESTADO DEMOCRÁTICO CUYAS POLÍTICAS PÚBLICAS LLEGUEN A TODO EL TERRITORIO}

Nuestro Estado, como la mayoría de los estados en América Latina no es democrático. Sus políticas sociales (educación y salud) no llegan a todos los peruanos y peruanas, en forma equitativa. Lo mismo sucede con la Ley, la justicia y la seguridad. La educación no llega a todo el territorio ni alcanza a todas las clases sociales. No todos asisten ni concluyen la 
secundaria. El $40 \%$ abandona sus estudios secundario y no logran por consiguiente, adquirir una de las herramientas que les permita romper el círculo vicioso de la pobreza. Algo similar sucede con el sector salud, medida en términos de número de médicos por habitante. El 79\% de las provincias no supera el mínimo de médicos señalado por el estándar internacional en salud. Como es obvio, las provincias de la costa son mejores atendidas tanto en la educación como en la salud, en desmedro de las provincias de la sierra y de la selva.

El Ministerio de Inclusión Social se propone democratizar el Estado con las siguientes medidas:

- El reconocimiento de los derechos civiles, políticos y sociales, de sus respectivas garantías para que las peruanas y los peruanos puedan ejercer efectivamente su ciudadanía.

-El despliegue de políticas sociales (salud, educación, justicia, seguridad) de calidad para todos.

- Creación del Estado móvil con la finalidad de articular sectorialmente las redes de educación, salud, nutrición, seguridad, justicia, infraestructura, agricultura, servicios sanitarios básicos y coordinar los niveles de gobierno para llevar el Estado a las zonas rurales del país a las que llega tarde, mal o nunca. La peculiaridad del Estado móvil es su desplazamiento en búsqueda de la población. El Estado va al encuentro de los ciudadanos para ponerse a su servicio particular, se le dará a las redes desplegadas en las fronteras.

- El establecimiento de los tambos para atender situaciones de emergencia.

\section{UNESTADO PROMOTORDELDESARROLLO}

El Estado y de sus políticas sociales en las diversas regiones y provincias del país. Se utilizaron diversos indicadores: Educación, salud, saneamiento (agua y desagüe) y electricidad, pero se dejaron de lado otras políticas, igualmente importantes (seguridad y justicia) probablemente porque son difíciles de cuantificar.

Si se relaciona la densidad del Estado con los ingresos familiares per cápita, se llega al siguiente resultado a más Estado, más ingresos familiares per cápita. Esto es, más desarrollo y a menos Estado, menos desarrollo. Por eso necesitamos fortalecer el Estado no solo para impulsar la inclusión, sino también, para promover el desarrollo. Una política que va en ese camino el gasto en el desarrollo de la ciencia y la tecnología.

El Perú ha cumplido a medidas esa tarea. El World Economic Forum, en su informe 2010 - 2011 presenta el índice de competitividad y propone como sus pilares el entorno del mercado, el entorno político y regulatorio, la infraestructura, la preparación individual, la disposición del gobierno, el uso individual, el uso comercial y el uso público.

La escasa productividad laboral promedio, que es diez veces inferior al valor mostrado por las economías desarrolladas según la OIT, obedece al limitado desarrollo de la ciencia, la tecnología y la innovación productiva. El indicador más representativo de este atraso en el Perú es el escaso número de patentes otorgadas a sus residentes, apenas quince frente a más de cien en países de la región como Argentina y México.

Esta situación se debe a que pese al mandato constitucional de promover el desarrollo científico y tecnológico del país, no hay políticas orientada a este sector. El rol del Estado es esencial por las externalidades positivas que genera el desarrollo tecnológico.

La propuesta se puede resumir en lo siguiente:

- Promover la investigación científica y tecnología proyectada a la innovación sobre la base de las prioridades del desarrollo y la inserción competitiva del Perú en la economía mundial.

- Propiciar la disminución de las brechas de conocimiento científico y tecnológico con los países industrializados.

- Asegurar un ambiente de competitividad, meritocracia y buenas prácticas de investigación en las universidades y centros de investigación del Estado.

- Promover las actividades profesionales de los investigadores científicos y tecnológicos que revaloren su papel y los orienten a la producción de conocimiento científico, tecnológico y de innovación para alcanzar estándares internacionales.

- Garantizar que el Sistema Nacional de Ciencia, Tecnología e Innovación, tecnologías se convierta 
en factor favorable para el desarrollo de la competitividad nacional.

- Promover el acercamiento de los centros de investigación de las universidades e instituciones públicas de investigación a las empresas, para realizar proyectos de investigación directamente vinculados con las necesidades del crecimiento económico.

- Impulsar la construcción de una cultura científica y tecnológica nacional que aliente la creatividad, la investigación científica, el desarrollo tecnológico y que favorezca la socialización y la apropiación de la ciencia, la tecnología y la innovación, con miras a ser parte de la sociedad del conocimiento.

- Reforzar los mecanismos para garantizar el derecho a la propiedad intelectual y la defensa del conocimiento tradicional.

- Fomentar la creación, modernización y permanente actualización de la infraestructura de investigación y desarrollo del país, en especial el establecimiento de parques científicos - tecnológicos y polos de innovación.

\section{UN EFECTIVO ESTADO DE DERECHO}

El Estado es una macro - estructura organizada para ejercer el dominio político y construir el orden legítimo. Cuando la Ley domina y limita a todos los elementos que integran el Estado, este se llama Estado de derecho. Este no es totalmente el caso peruano. Normalmente tenemos un Estado de derecho, pero no es efectivo en la medida que la Ley no llega realmente a todo el territorio ni a todos los peruanos por igual.

La vigencia del Estado de derecho es una tarea que atañe a todos los poderes del Estado, pero es una función específica del poder judicial y del poder legislativo. En lo que atañe al poder ejecutivo proponemos las siguientes medidas:

- Respeto a la constitución y apoyo a la reforma de la misma, por los canales que ella establece.

-Establecimiento del dominio efectivo de la Ley en todo el territorio.

- Impulso a la justicia igual para todos.

\section{UN ESTADO QUE GARANTICE LA SEGURIDAD EXTERNA Y SEGURIDAD CIUDADANA}

Un Estado no está condicionado solo por la dinámica interna de una sociedad, sino también, por el Sistema de Estados en el que se ubica. Según el sociólogo Charles Tilly todos los estados han sido construidos por los guerreros (Prusia, Rusia, Eslovenia) y los empresarios (Holanda, Las ciudades - Estado Italianas) o por la alianza, entre ellos (Inglaterra, Irlanda, Escocia). No podemos dejar de lado, este dato de la historia que ha tenido en nuestro caso experiencias dramáticas. Tenemos por eso que garantizar nuestra seguridad externa e interna, la seguridad nacional y la seguridad ciudadana.

\section{a. Seguridad nacional}

En el frente externo desplegar las respuestas necesarias y equilibradas para responder a las amenazas a la soberanía nacional.

En el frente interno prevenir e intervenir en aquellos conflictos que amenazan la gobernabilidad del país.

\section{b. Seguridad ciudadana}

Establecer las coordinaciones necesarias entre las comisarías, los municipios y la participación ciudadana para garantizar la seguridad de todos.

\section{UN ESTADO UNITARIO QUE RECONOCE LA PLURICULTURALIDAD DEL PAÍS}

El Perú es un país multicultural complejo. Es, principalmente, multiétnico y a la vez, multinacional, a diferencia de Bolivia y Ecuador, los quechuas y los aymaras del Perú no demandan un Estado multinacional sino una mejor forma de integración al país.

Las políticas que buscan plasmar esta aspiración son las siguientes:

- Desarrollar las políticas de salud, educación, justicia, seguridad en la segunda de origen.

- Fomentar el respecto a su cultura.

- Reconocer sus derechos.

- Ejecutar políticas que reconozcan los derechos 
colectivos de las etnias de la selva: derecho a la diferencia, autonomía del territorio que ocupan y formas de representación propia.

\section{UN ESTADO DESCENTRALIZADO}

La descentralización es una política de Estado que permitirá fortalecer la unidad de la nación, democratizado el poder, potenciando los diversos territorios y sus ámbitos locales y regionales $y$, dinamizando los 5 grandes espacios territoriales que se configuran en el país: el marco norte; el amazónico; el marco sur; el centro sur y el de Lima-Callao. Afirmamos nuestro objetivo nacional de ser el país de macro regiones transversales y que la descentralización sea el reencuentro con las raíces y potencialidades territoriales de la nación, para alcanzar nuestro objetivo nacional bicentenario.

Es urgente ordenar el territorio y su gestión, pues el desorden existente al respecto, es uno de los factores de deterioro ambiental y de crisis social.

Se orienta los próximos años, se institucionalizará los instrumentos de gestión del territorio para elaborar en forma participativa los planes regionales y provinciales de ordenamiento territorial. Para lograr estos objetivos se proponen las siguientes medidas.

- Impulso de políticas de igualación entre las regiones estableciendo un fondo de compensación regional.

- Promoción de los mercados regionales mediante Alianza Público - Privadas.

-Establecimiento de la descentralización fiscal.

- Reordenamiento del territorio en macro regiones $y$ en mancomunidades.

Finalmente, el Estado está obligado a dedicar una parte significativa de la presión tributaria a las políticas sociales universales (salud, educación, justicia y seguridad) de calidad para todos, y a las políticas sociales temporales, solo si ellas llegan a todo el territorio y a todas las clases sociales, la sociedad le otorgará legitimidad al Estado, al gobierno y a los gobernantes. En el fondo, las relaciones funcionales señaladas constituyen un compromiso entre los empresarios y los trabajadores con la mediación efectiva del Estado. Se trata, en última instancia, de construir no solo un Estado democrático sino un capitalismo democrático, las medidas para fortalecer las finanzas del Estado son las siguientes:

- El gravamen a la explotación minera.

- Formalización a la economía informal.

- Combate a la evasión tributaria.

- Combate a la elusión tributaria.

\section{UN ESTADO EFICAZ, EFICIENTE Y CREÍBLE}

El gobierno del Presidente actual se orienta profundizar la reforma del servicio civil, a través de la autoridad del servicio civil (servir), mejorando el desempeño sobre la base de los principios de mérito, flexibilidad, igualdad de oportunidades e imparcialidad para atender mejor al ciudadano.

Los objetivos de la reforma en materia de servicio civil son:

- Profesionalizar el servicio civil estableciendo un sistema basado en el reclutamiento meritocrático, generando las condiciones necesarias para atraer y retener a personas idóneas para el servicio civil.

- Ordenar los derechos y deberes de los servidores públicos, reduciendo las distorsiones en responsabilidades y remuneraciones, atendiendo a la naturaleza y características a cada grupo particular (directivos de confianza, profesionales y de apoyo).

- Promover la gestión por resultados, generando incentivos por logros y no por cumplimiento de procesos y orientando la gestión a mejorar la atención y el servicio del ciudadano.

- Generar las capacidades del Estado para la gestión moderna de las oficinas de recursos humanos o de gestión de personas.

- Generar un sistema de evaluación desempeño y de desarrollo de capacidades.

\section{UN ESTADO Y UN GOBIERNO TRANSPARENTE}

Desde una perspectiva comparada, el Perú es percibido como uno de los países latinoamericanos más corruptos juntamente con Guatemala, Nicaragua, Jamaica y Ecuador, como lo señala una reciente publicación de IEP sobre la cultura política de la 
democracia en el Perú. Las principales medidas de lucha contra la corrupción son las siguientes:

-Establecimiento estricto de la rendición de cuentas en todas las instituciones del Estado.

- Exigencia de las normas de transparencia a todas las instituciones del Estado.

- Coordinación de los órganos de control horizontal o institucional con los medios y la sociedad civil en la lucha contra la corrupción.

- Nombramiento y control del contralor por el congreso.

\section{ECONOMÍA COMPETITIVIDAD Y EMPLEO}

En el Perú entre 1998 y 2010 ha descendido el porcentaje de subempleo y desempleo respecto del total de la PEA. El subempleo bajó de 50\% a 46\% de la PEA, mientras el desempleo se redujo del $6 \%$ al $4 \%$ de la PEA. Sin embargo, sigue siendo indispensable que baje todavía más. A su vez, el Perú ha mejorado su competitividad, pero mantiene como debilidad persistente un bajo índice de productividad per cápita. Actualmente, el 68\% de la PEA trabaja en empresas de no más de cinco trabajadores en condiciones técnicas y productivas inferiores al promedio latinoamericano.

Los factores claves asociados al crecimiento sostenido del PBI con alto contenido de empleo son: la inversión, la productividad del trabajo, el capital humano (educación superior, porcentaje de empleo industrial), el capital natural, el tamaño del mercado interno y la participación en el comercio internacional.

La economía necesita alcanzar determinadas condiciones para generar mayores tasas de crecimiento sostenible, pero que a la vez hagan posible el aumento de empleos de calidad y terminan de derrotar a la pobreza la agrupación de los sectores productivos en primarios, secundarios y terciarios permite apreciar el relativo bajo nivel de las actividades de transformación y creación de mayor valor agregado (secundarias) en la estructura económica del Perú, las que juntamente con los servicios generan la mayor cantidad de fuentes de trabajo. Esta estructura ha variado relativamente desde 1975, a pesar de que hacia ese año la participación de los sectores secundarios se había incrementado, mientras que las actividades del sector primario se habían reducido.

\section{EJES DE DESARROLLO}

\section{a. Objetivo nacional}

El objetivo es lograr una economía dinámica y diversificada, integrada competitivamente a la economía mundial y con un mercado interno desarrollado, en un marco de reglas estables que promuevan la inversión privada con alta generación de empleo y elevada productividad del trabajo.

Durante la recuperación del Sistema Económico Internacional se acondicionarán, como parte de las políticas de mejoramiento de la competitividad internacional, la infraestructura, la lógica y el capital humano, haciendo uso intensivo del empleo y de insumos nacionales para mantener la demanda efectiva interna. Ello, permitirá que las exportaciones tiendan a un nivel superior, con mayor valor agregado e incorporación de conocimiento.

El impulso a las actividades generadoras de empleo es clave para la ampliación del mercado interno y el desarrollo de actividades productivas orientadas a este, las que por su competitividad también poseerán un potencial exportador.

De este modo, se busca conformar una estructura productiva moderna, con fuertes eslabonamientos hacia atrás y hacia delante, desplegada equilibradamente a nivel regional, y en la que la fuerza laboral, gracias a la distribución del ingreso, tenga acceso a los mercados de bienes y servicios.

\section{b. Lineamientos de política}

\section{Política económica}

- Asegurar la credibilidad y predictibilidad de la política económica, garantizando la estabilidad moderna y de precios, así como, de las reglas de juego para la inversión.

- Fortalecer la política y la estrategia de apertura comercial mediante la diversificación hacia los nuevos mercados emergentes y en el marco de los acuerdos comerciales.

- Promover la inversión pública y privada, nacional y extranjera, asegurando su efecto multiplicador 
en el país en forma concertada y garantizando su seguridad.

- Reducir la vulnerabilidad de la economía frente a eventos externos mediante la expansión del mercado interno.

- Adoptar medidas para ampliar la base tributaria, evitando sobrecargar las actividades económicas formales y su regresividad.

- Simplificary controlar permanentemente el marco legal, la estructura y la administración tributaria.

- Incentivar el desarrollo descentralizado.

- Facilitar y apoyar los procesos de financiamiento de proyectos de inversión pública a cargo de los gobiernos regionales.

\section{Estructura productiva}

- Promover la articulación de las empresas exportadoras con las industrias de insumos, bienes de capital y servicios, con miras al desarrollo de actividades conexas de alto nivel tecnológico y valor agregado relacionadas con los recursos naturales estratégicos del mar territorial y de la costa, sierra y selva.

- Impulsar la inversión en infraestructura logística y productiva local y regional pública y privada.

- Promover la producción, el desarrollo empresarial local y el empleo mediante el impulso al desarrollo de industrias de transformación, priorizando los sectores de producción exportable.

- Fortalecer las industrias nacionales orientadas al mercado interno y promover su participación en los mercados internacionales.

- Estimular la exploración y explotación minera con el enfoque de responsabilidad social, ambiental y sustentable.

- Concertar con las empresas mineras la transformación industrial de su producción, para incrementar el valor agregado preferentemente en el lugar de explotación.

- Apoyar a la investigación y desarrollo para aplicaciones de la producción minera en aleaciones ligeras para la microelectrónica y la robótica.

- Promover el desarrollo de los servicios turísticos y de gastronomía, así como, las actividades vinculados a estos.

- Posibilitar el acceso de todos los tipos de empresas, en especial de las MYPE, a los mercados financieros con igualdad de oportunidades y promover el desarrollo empresarial en la conducción de las unidades de producción familiar en los ámbitos urbano y rural.

- Regular y supervisar los monopolios para evitar el abuso de la posición de dominio.

- Apoyar la transformación de la formación profesional universitaria estatal para alcanzar la calidad y competitividad internacionales y hacerla concordante con la modernización productiva.

- Mantener mecanismos de diálogo y coordinación permanentes entre las distintas entidades del sector público y el sector privado, para definir temas estratégicos de desarrollo e instrumentos que permitan mejorar y consolidar la competitividad del sector productivo.

\section{Competitividad e integración a los mercados globales}

- Promover el aprovechamiento de las ventajas de los acuerdos y tratados comerciales con Estados Unidos, la Unión Europea, el APEC, la CAN y el MERCOSUR.

-Estimular la producción exportable competitiva con alto valor agregado.

- Impulsar las exportaciones de productos ecológicos con el fin de incrementar su participación en el valor de las exportaciones.

- Promover el uso de las tecnologías de información como forma de reducir costos, ampliar mercados y mejorar la competitividad.

- Impulsar la integración física, comercial y económica con Brasil.

\section{Innovación y Tecnología}

- Promover la investigación científica y tecnológica proyectada a la innovación con base a las prioridades del desarrollo y la inserción competitiva del Perú en la economía mundial.

- Propiciar la disminución de las brechas de cono- 
cimiento científico y tecnológico con los países industrializados.

- Asegurar un ambiente de competitividad, meritocracia y buenas prácticas de investigación en las universidades y centros de investigación del Estado.

- Promover las actividades profesionales de los investigadores científicos y tecnológicos que revaloren su papel y los orienten a la producción de conocimiento científico, tecnológico y de innovación para alcanzar estándares internacionales.

- Garantizar que el Sistema Nacional de Ciencia Tecnología e Innovación Tecnológica se convierta en factor favorable para el desarrollo de la competitividad nacional.

- Promover el acercamiento de los Centros de Investigación de las Universidades e Instituciones Públicas de Investigación a las empresas, para realizar proyectos de investigación directamente vinculadas con las necesidades del crecimiento económico.

- Impulsar la construcción de una cultura científica y tecnológica nacional que aliente la creatividad, la investigación científica, el desarrollo tecnológico y que favorezca la socialización y la apropiación de la ciencia, la tecnología y la innovación, con miras a ser parte de la sociedad del conocimiento.

- Fomentar la creación, modernización y permanente actualización de la infraestructura de investigación y desarrollo del país.

- Promover la co - responsabilidad pública y privada en el financiamiento de las actividades de ciencia, tecnología e innovación a nivel nacional y regional.

- Impulsar el establecimiento de un Sistema Nacional de Información de Ciencia, Tecnología e Innovación que sea incluyente y descentralizado.

- Reforzar los mecanismos para garantizar el derecho a la propiedad intelectual y la defensa del conocimiento tradicional.

\section{Empleo}

- Promover la modernización de las unidades productivas familiares y reducir la informalidad urbana y la agricultura de subsistencia mediante el establecimiento de complejos integrados de producción e incubadoras de empresas, con el apoyo sostenido de los gobiernos regionales y locales.

- Impulsar la competitividad laboral de los trabajadores a estándares internacionales mediante la concertación entre el Estado, la empresa privada y los propios trabajadores.

- Promover el acceso de las mujeres, los jóvenes, los adultos mayores y los discapacitados a los mercados de trabajo.

- Apoyar la competitividad empresarial de las MYPE, las PYME y la agricultura campesina, y promover una normativa que establezca un marco legal e institucional para que estas unidades económicas funcionen como tercer sector.

- Promover la capacitación para el trabajo, la reconversión laboral y la formación continua en las empresas, así como la orientación vocacional, la información ocupacional y la normalización y certificación de competencias laborales para desarrollar los recursos humanos.

\section{Acciones estratégicas}

- Apoyar la inserción de bienes y servicios peruanos de manera competitiva en los mercados internacionales.

- Mejorar las condiciones de acceso a los mercados y establecer reglas y disciplinas claras para el intercambio de bienes, servicios e inversiones.

- Posicionar una "marca de país" a nivel internacional, asociada a las ventajas competitivas del Perú y sus productos emblemáticos, a fin de favorecer la apertura de nuevos mercados para la producción nacional.

- Fortalecer el sistema de inteligencia comercial.

- Promover iniciativa de bio comercios articulados con mercados especializados de alto valor.

- Incrementar la participación de los productos ecológicos en las exportaciones peruanas. 


\section{CONCLUSIONES}

1. La descentralización es el proceso mediante el cual se trata de promover y llevar a cabo las transferencias de competencias económicas, fiscales, administrativas y sociales por parte del gobierno central a los gobiernos regionales y locales, pretendiendo con ello, materializar un conjunto de reglas unificadoras que produzcan la capacidad institucional del sistema de relaciones intergubernamentales. Dicho en otras palabras, la descentralización es una dinámica social y política de gran complejidad, en la cual confluyen diversos factores económicos, fiscales, políticos institucionales y culturales.

2. Al ser compleja, la descentralización implica varias dimensiones, etapas o niveles que deben ser atendidas y relacionada de manera coherente con la realidad de la población peruana, en base a ello tenemos: una dimensión social, que implica el fortalecimiento de diversas formas de Asociación Ciudadana para que la población puede ejercer plenamente sus derechos democráticos, una dimensión ambiental, la misma, que implica tomar conciencia de los diversos recursos naturales y actividades productivas de la región, para que sus gobiernos regionales y locales hagan un uso adecuado de estos, fomentando así el desarrollo sostenible del país y por último, se quiere una dimensión política educativa que construya la formación de su población para hacerla más productiva y comprometida con su realidad.

3. La descentralización por sí sola no es sinónimo de desarrollo, sino, más bien tiene que complementarse con una serie de medidas económicas, sociales, culturales, que le tienen que anteceder para que en conjunto se logre un desarrollo mancomunado y duradero; por ello que, para descentralizar nuestro país se tendría que seguir pasos concretos que sirvan a largo plazo para la eficiente administración del Estado, dichos pasos a seguir se pueden materializar, en los siguientes enunciados:

- Como primordial, medida a paso, es tener un país totalmente comunicado, cosa que el Perú carece.
- Segundo paso, vendría a ser el desarrollo de las ciudades que puedan convertirse en núcleos verdaderos de cohesión de regiones económicamente aislados.

4. La descentralización desde el punto de vista político, se centra en el inherente derecho democrático a la autonomía tanto de los gobiernos regionales como de las municipalidades y el costo fiscal del gobierno central para asegurarlo. En base a esta perspectiva, se debe de tener en cuenta que las regiones y municipalidades quieren mayor autonomía institucional, aunque con mayores niveles de dependencia fiscal en donde debe primar la transparencia por parte del gobierno central. En esta línea de pensamiento la descentralización ejerce su efecto, al dotar tanto a las regiones como a los municipios de autonomía para manejar y controlar los intereses que le fueron confiados que se generan dentro de su jurisdicción.

\section{REFERENCIAS BIBLIOGRÁFICAS}

1. Asamblea Nacional de Gobierno Regionales (ANGR). ( 2010) Documentos de debate No 4 Propuesta Técnico Legal de Descentralización Fiscal. Lima.

(2011) Documento de debate No 5 Aportes de los Gobiernos Regionales en el marco del proceso de Descentralización (2006-2009) Lima.

2. AZPUR, Javier (2010) Aportes para un necesario balance de la reforma descentralista en: centralismo y concentración. Serie: Perú Hoy No 18 Lima: Centro de Estudios y Promoción del Desarrollo (DESCO).

3. BALLÓNECHEGARAY,Eduardo (2008) Balance del proceso peruano de descentralización desde los gobiernos regionales Lima. CIES.

4. Banco Mundial (2011) Perú en el umbral de una nueva era lecciones y desafíos para consolidar el desarrollo económico y un modelo más incluyente.

5. CASAS TRAGODARA, Carlos (2009) Descentralización Fiscal frente a un contexto rural. Lima: Red de Municipalidades Rurales del Perú. (2009) Propuestas para avanzar en la Descentra- 
lización Fiscal en el Perú Sistematización de Estudios Lima.

6. GARCIA NARANJO MORALES Aida (2008) Políticas públicas y equidad de género. Lima. Centro de Asesoría Laboral del Perú. CEDAL.

7. HIRSCHMAN. Albert. (1916) La estrategia del Desarrollo Económico. México Fondo de Cultura Económica FCE.

8. SANTA CRUZ, J. Francisco (2007) La Regionalización y el fortalecimiento de las Juntas de Coordinación Interregional Serie: Cuadernos Descentralistas No 21. Lima.
9. TANAKA, Martín (2007) La Participación Ciudadana en el Sistema Representativo. Lima (PRODES).

10. TELLO, Mario (2008) Desarrollo Económico Local. Descentralización y Clusters. Teoría, Evidencias y Aplicaciones CENTRUM Católica. Lima.

11. VEGA, Jorge (2008) Análisis del Proceso de Descentralización Fiscal en el Perú. Lima. CIES. 